\title{
Perfil Aterosclerótico da Artéria Carótida como Preditor de Risco para Reestenose após Implante de Stent Coronário
}

\author{
Carotid Artery Atherosclerotic Profile as Risk Predictor for Restenosis After Coronary Stenting \\ Cássia da Silva Antico Rodrigues, ${ }^{1}$ Rodrigo Bazan, ${ }^{1}$ Fabrício Moreira Reis, ${ }^{1}$ Caroline F. S. Mazeto Pupo da Silveira, ${ }^{1}$ \\ Lívia Maria Severino Hueb, ${ }^{1}$ Fábio Cardoso de Carvalho, ${ }^{1}$ Hélio Rubens de Carvalho Nunes, ${ }^{1}$ Katashi Okoshi, ${ }^{10}$ \\ João Carlos Hueb, ${ }^{1}$ Silméia Garcia Zanati Bazan ${ }^{10}$ \\ Universidade Estadual Paulista Julio de Mesquita Filho - Faculdade de Medicina Campus de Botucatu, ${ }^{1}$ Botucatu, SP - Brasil
}

\section{Resumo}

Fundamento: A incidência de reestenose da artéria coronária após o implante de um stent não farmacológico é mais baixa que na angioplastia com balão; no entanto, ainda apresenta altas taxas.

Objetivo: $\mathrm{O}$ objetivo deste estudo foi identificar novos indicadores de risco para reestenose de stent usando ultrassonografia das carótidas que, em conjunto com indicadores já existentes, ajudariam na escolha do stent.

Métodos: Realizamos um estudo prospectivo transversal incluindo 121 pacientes consecutivos com doença arterial coronariana que foram submetidos à intervenção coronária percutânea com angiografia nos 12 meses anteriores. Após os casos de reestenose de stent serem identificados, os pacientes foram submetidos à ultrassonografia de carótidas para avaliar a espessura da camada íntima média e placas ateroscleróticas. Os dados foram analisados por regressão múltipla de Cox. O nível de significância foi $\mathrm{p}<0,05$.

Resultados: A idade mediana dos pacientes foi de 60 anos (10 quartil $=55,3^{\circ}$ quartil $=68$ ), e $64,5 \%$ dos pacientes eram do sexo masculino. A angiografia coronária mostrou que 57 pacientes $(47,1 \%)$ apresentaram reestenose de stent. Cinquenta e cinco pacientes (45,5\%) apresentaram placas ateroscleróticas ecolucentes nas artérias carótidas e 54,5\% apresentaram placas ecogênicas ou nenhuma placa. Dos pacientes que apresentaram placas ecolucentes, 90,9\% apresentaram reestenose do stent coronário, e daqueles com placas ecogênicas ou nenhuma placa, 10,6\% apresentaram reestenose de stent. A presença de placas ecolucentes nas artérias carótidas aumentou o risco de reestenose de stent coronário em 8,21 vezes $(R R=8,21 ; I C 95 \%: 3,58-18,82 ; p<0,001)$.

Conclusões: A presença de placas ateroscleróticas ecolucentes na artéria carótida constitui um preditor de risco de reestenose de stent coronário e deve ser considerada na escolha do tipo de stent a ser usado na angioplastia coronária. (Arq Bras Cardiol. 2021; 116(4):727-733)

Palavras-chave: Doença Arterial Coronariana; Aterosclerose; Reestenose Coronária; Stents; Angioplastia Coronária com Balão; Artérias Carótidas/ultrassonografia; Placa Aterosclerótica.

\begin{abstract}
Background: The incidence of restenosis of the coronary artery after a bare-metal stent implant has been lower than in simple balloon angioplasty; however, it still shows relatively high rates

Objective: The aim of this study was to find new risk indicators for in-stent restenosis using carotid ultrasonography, that, in addition to the already existing indicators, would help in decision-making for stent selection.

Methods: We carried out a cross-sectional prospective study including 121 consecutive patients with chronic coronary artery disease who had undergone percutaneous coronary intervention with repeat angiography in the previous 12 months. After all cases of in-stent restenosis were identified, patients underwent carotid ultrasonography to evaluate carotid intima-media thickness and atherosclerosis plaques. The data were analyzed by Cox multiple regression. The significance level was set a $p<0.05$
\end{abstract}

Results: Median age of patients was 60 years (1st quartile $=55$, 3rd quartile $=68$ ), and $64.5 \%$ of patients were male. Coronary angiography showed that 57 patients (47.1\%) presented in-stent restenosis. Fifty-five patients (45.5\%) had echolucent atherosclerotic plaques in carotid arteries and $54.5 \%$ had echogenic plaques or no plaques. Of patients with who had echolucent plaques, 90.9\% presented coronary in-stent restenosis. Of those who had echogenic plaques or no plaques, $10.6 \%$ presented in-stent restenosis. The presence of echolucent plaques in carotid arteries increased the risk of coronary in-stent restenosis by 8.21 times ( $R R=8.21 ; 95 \% \mathrm{Cl}: 3.58-18.82 ; \mathrm{p}<0.001)$.

Conclusions: The presence of echolucent atherosclerotic plaques in carotid artery constitutes a risk predictor of coronary in-stent restenosis and should be considered in the selection of the type of stent to be used in coronary angioplasty. (Arq Bras Cardiol. 2021; 116(4): 727-733)

Keywords: Coronary Artery Disease; Atherosclerosis; Coronary restenosis; Stents; Angioplasty, Balloon, Coronary; Carotid Arteries/ultrasonography; Plaque, Atherosclerotic.

Full texts in English - http://www.arquivosonline.com.br

Correspondência: Silméia Garcia Zanati Bazan •

Universidade Estadual Paulista Julio de Mesquita Filho Faculdade de Medicina Campus de Botucatu - Distrito Rubião Jr, s/n. CEP 18618-687, Botucatu, SP - Brasil E-mail: sgz.bazan@unesp.br

Artigo recebido em 07/01/2019, revisado em 20/09/2019, aceito em 27/12/2019

DOI: https://doi.org/10.36660/abc.20190650 


\section{Introdução}

O desenvolvimento de stents não farmacológicos (SNF) foi um avanço importante na angioplastia com balão para o tratamento de doença arterial coronariana sintomática. Com o uso de stents, pode-se evitar a reestenose por meio da atenuação da retração elástica e remodelamento geométrico negativo, resultando na redução do lúmen do vaso. ${ }^{1}$ Contudo, a necessidade de novas revascularizações devido a reestenoses de stent ainda era alta, ocorrendo em $10-20 \%$ dos pacientes, causadas principalmente por crescimento excessivo da neoíntima, muitas vezes superior à hiperplasia intimal observada na angioplastia simples com balão. ${ }^{2,3}$

Mais recentemente, os stents farmacológicos (SF) foram desenvolvidos para reduzir a elevada taxa de reestenose observada com os SNF e a necessidade de revascularização. Ensaios clínicos confirmaram uma redução de 50\% a 70\% na necessidade de revascularizações da lesão alvo com o uso de SF em comparação a SNF, apesar de não ter sido observada diferença significativa na taxa de mortalidade global entre eles. ${ }^{4-9}$ Esses resultados levaram à recomendação do uso de SF na intervenção coronária percutânea. Porém, esses stents são de alto custo e exigem um longo período de terapia antiplaquetária dupla para prevenir a ocorrência de trombose, não sendo recomendados para todos os pacientes. ${ }^{10}$

Em algumas situações, tais como diabetes mellitus, envolvimento de pequenos vasos, stent dentro de outro stent, lesões de bifurcação, lesões extensas ou múltiplas, e enxerto de veia safena, a angioplastia com implante de stent apresenta alto risco de reestenose (30-60\%). Nessas condições, os SF são mais indicados. ${ }^{11}$

Além das situações mencionadas, pouco se sabe sobre a importância das placas ateroscleróticas nas artérias carótidas e sua correlação com reestenose de stent. ${ }^{12}$

Tal correlação é possível, uma vez que inflamação é comum em ambos os casos. Segundo Corrado et al., ${ }^{13} \mathrm{em}$ pacientes submetidos ao implante de stent coronário, observa-se uma maior frequência de reestenose de stent em pacientes com maior espessura da camada íntima-média (EIM) da parede da carótida e mais placas ateroscleróticas nas artérias carótidas.

Uma vez que a maioria dos indicadores de risco para reestenose de stent refere-se a aspectos angiográficos, o objetivo do estudo foi correlacionar, usando-se ultrassonografia, o perfil aterosclerótico da artéria carótida com reestenose de stent coronário, com ênfase na presença de placas ecolucentes.

\section{Pacientes e métodos}

\section{Pacientes}

O estudo foi aprovado pelo comitê de ética da Faculdade de Medicina de Botucatu. Todos os pacientes assinaram o termo de consentimento antes de participarem do estudo. Conduzimos um estudo prospectivo transversal incluindo 121 pacientes consecutivos com doença arterial coronariana crônica entre fevereiro e dezembro de 2015. Todos os pacientes haviam sido submetidos à intervenção coronária percutânea e outra angiografia dentro de 12 meses. As angiografias foram indicadas para estratificação de risco de angina, ou realizadas após confirmação de isquemia miocárdica em testes "provocativos" (teste de esforço com exercício ou cintilografia do miocárdio com estresse). Com base na entrevista direta, identificamos quais pacientes apresentaram diabetes mellitus, dislipidemia ou hipertensão arterial. Também identificamos se os pacientes eram fumantes, e quais medicamentos usavam. A angiografia coronária detectou implantação de stent prévia nas artérias coronárias (artéria coronária direita, artéria coronária circunflexa, artéria coronária descendente anterior, e seus respectivos ramos).

\section{Ultrassom com Doppler das Carótidas}

Todos os exames foram realizados por um ultrassonografista usando um aparelho Vivid S6 (General Electric Medical Systems, Tirat Carmel, Israel) equipado com um transdutor linear de frequência $8 \mathrm{MHz}$. O ultrassom da artéria carótida com Doppler foi realizado com o paciente na posição supina. As imagens da carótida, incluindo espessura da íntima-média foram analisadas conforme determinado nos consensos da Sociedade Americana de Ecocardiografia (American Society of Echocardiography) ${ }^{14} \mathrm{e}$ de Mannheim, ${ }^{15}$ e registradas e armazenadas em um CD. A classificação da EIM por sexo e idade foi feita com base nos valores do percentil 75 propostos no estudo CAPS. ${ }^{16}$

A EIM foi medida utilizando-se um padrão de linha dupla visualizado por ecotomografia em ambas as artérias carótidas comuns em uma imagem longitudinal. Esse padrão de linha dupla corresponde às extremidades das interfaces lúmen-íntima e média-adventícia. Os valores médios foram calculados em um segmento de $10 \mathrm{~mm}$ próximo à parede posterior do bulbo carotídeo. A placa foi considerada quando uma estrutura focal invadisse o lúmen arterial em pelo menos 0,5mm, ou correspondesse a 50\% do valor da EIM circundante, ou quando a medida da espessura fosse maior que 1,5 mm, medida desde a interface média-adventícia à interface lúmen-íntima. As placas foram descritas segundo classificação de Gray-Weale et al.,17 Em resumo, placas tipo 1 são aquelas uniformemente ecolucentes; placas tipo II são predominantemente ecolucentes; placas tipo III são placas predominantemente ecogênicas; e as placas tipo IV são uniformemente ecogênicas. Para a análise estatística, os tipos I e II foram chamadas ecolucentes e as tipo III e IV ecogênicas.

\section{Angiografia coronária}

As angiografias coronárias foram realizadas por cateterismo cardíaco transradial. Após angiografia coronária seletiva e identificação do stent, a reestenose foi avaliada por angiografia quantitativa. Reestenose de stent foi definida como redução do lúmen de $50 \%$ ou mais. ${ }^{18,19}$

\section{Análise estatística}

As variáveis contínuas foram apresentadas como medianas e valores mínimos e máximos. As variáveis categóricas foram expressas como valores absolutos ou frequência (\%). A análise de preditores de risco para reestenose de stent em 12 meses de seguimento foi realizada em duas etapas. $\mathrm{Na}$ etapa 1, foi estimado o risco relativo individual para cada preditor potencial; na fase 2, o modelo de regressão múltipla de Cox foi ajustado para o risco de reestenose de stent com 
os preditores mais fortemente associados $(p<0,05)$ com reestenose detectados na fase 1 . Valores de $p<0,05$ foram considerados estatisticamente significantes. Todas as análises estatísticas foram realizadas usando o SPSS v21.0.

\section{Resultados}

A idade mediana dos 121 pacientes foi 60 anos (10 quartil $=55 ; 3$ o quartil $=68) ; 78$ pacientes $(64,5 \%)$ eram do sexo masculino. Cinquenta e oito $(47,9 \%)$ dos pacientes eram fumantes, 47 (38,8\%) diabéticos, 91 (75,2\%) apresentaram hipertensão sistêmica, e 119 (98,3\%) apresentaram dislipidemia. Após ajuste do modelo de regressão múltipla de Cox quanto ao risco de reestenose de stent por potenciais preditores, observamos que não houve diferença estatisticamente significante na distribuição dessas variáveis nos subgrupos com ou sem reestenose de stent (Tabela 1).

Os locais de stent foram: artéria descendente anterior esquerda (ADA) em 50 pacientes (41,3\%); artéria coronária direita (ACD) em 34 pacientes (28,1\%); artéria circunflexa esquerda (ACE) em 19 pacientes (15,7\%), ADA e ACD em 9 pacientes (7,4\%), ADA e ACE em 5 pacientes (4,1\%), e ACD e ACE em 4 pacientes (3,3\%). As angiografias mostraram que 57 pacientes $(47,1 \%)$ apresentaram reestenose de stent coronário, e o local do stent não influenciou na taxa de reestenose de stent (Tabela 1).

A maioria dos pacientes tomava aspirina $(97,5 \%)$, estatina $(92,6 \%)$, inibidores da enzima conversora de angiotensina ou bloqueadores de receptor de angiotensina (80,2\%), betabloqueadores $(88,4 \%)$, e $27,3 \%$ dos pacientes usavam clopidogrel.

Cinquenta e cinco pacientes $(45,5 \%)$ dos pacientes apresentaram placas ecolucentes nas artérias carótidas e 66 pacientes (54,5\%) apresentaram placas ecogênicas ou nenhuma placa. Cinquenta (90,9\%) pacientes com placas ecolucentes, e somente sete $(10,6 \%)$ daqueles com placas ecogênicas ou nenhuma placa apresentaram reestenose de stent (Figura 1).

As imagens de ultrassonografia das placas carótidas e os achados angiográficos das artérias coronárias são apresentadas nas Figuras 2 e 3, respectivamente.

A análise de regressão múltipla revelou que a presença de placas ecolucentes nas artérias carótidas aumentou o risco de reestenose de stent coronário em 8,21 vezes $(8,21$; IC95\% 3,58$18,82 ; p<0,001)$. No entanto, observamos que uma EIM elevada não aumentou o risco de reestenose de stent coronário (RR 1,03; IC95\% 0,60-1,76; $p=0,897$ ).

\section{Discussão}

O estudo revelou uma clara correlação entre placas ateroscleróticas ecolucentes nas artérias carótidas e reestenose de stent coronário avaliado 12 meses após o implante do stent. Pacientes com placas ecolucentes nas artérias carótidas apresentaram um risco 8,21 vezes maior de reestenose de stent coronário em comparação àqueles com placas ecogênicas ou nenhuma placa nas carótidas. Um estudo prévio, no entanto, relatou uma correlação entre placas ecolucentes e reestenose de stent coronário com um OR de $3,8 .{ }^{20}$ Apesar de considerados obsoletos muitas vezes, SNF foram usados em ambos os estudos, o que reflete a realidade na América Latina. Apesar de similares, os estudos diferem-se na etnicidade das populações e no segundo agente antiplaquetário empregado, uma vez que o estudo de 2008 usou ticlopidina. Uma possível justificativa para essa correlação é inflamação, comum a ambas as situações. Os macrófagos foram as primeiras células inflamatórias a serem reconhecidas como associadas à aterosclerose. ${ }^{21}$ Posteriormente, outros tipos de leucócitos relacionados à inflamação tais como monócitos, neutrófilos, e linfócitos

Tabela 1 - Risco de reestenose de stent estimado para cada variável

\begin{tabular}{|c|c|c|c|}
\hline Variável & $\mathbf{R} \mathbf{R}$ & IC95\% & $\mathbf{p}$ \\
\hline Idade & 0,99 & $0,96-1,02$ & 0,555 \\
\hline Sexo masculino & 1,87 & $1,01-3,46$ & 0,048 \\
\hline \multicolumn{4}{|l|}{ História clínica } \\
\hline $\mathrm{HA}$ & 0,85 & $0,47-1,51$ & 0,567 \\
\hline DM & 1,07 & $0,63-1,81$ & 0,815 \\
\hline Tabagismo & 1,21 & $0,72-2,03$ & 0,478 \\
\hline Dislipidemia & 0,94 & $0,13-6,80$ & 0,952 \\
\hline \multicolumn{4}{|l|}{ US artérias carótidas } \\
\hline Placas ecolucentes & 8,57 & $3,89-18,90$ & $<0,001$ \\
\hline EIM (aumentada) & 1,88 & $1,11-3,15$ & 0,017 \\
\hline \multicolumn{4}{|l|}{ Coronária com stent } \\
\hline ADA & 1,23 & $0,72-2,07$ & 0,450 \\
\hline ACD & 0,76 & $0,44-1,32$ & 0,330 \\
\hline ACE & 0,85 & $0,45-1,60$ & 0,607 \\
\hline
\end{tabular}

HA: hipertensão arterial; DM: diabetes mellitus; US: ultrassonografia; EIM: espessura da camada intima-média; ADA: artéria descendente anterior esquerda; $A C D$ : artéria coronária direita; $A C E$ : artéria circunflexa esquerda 


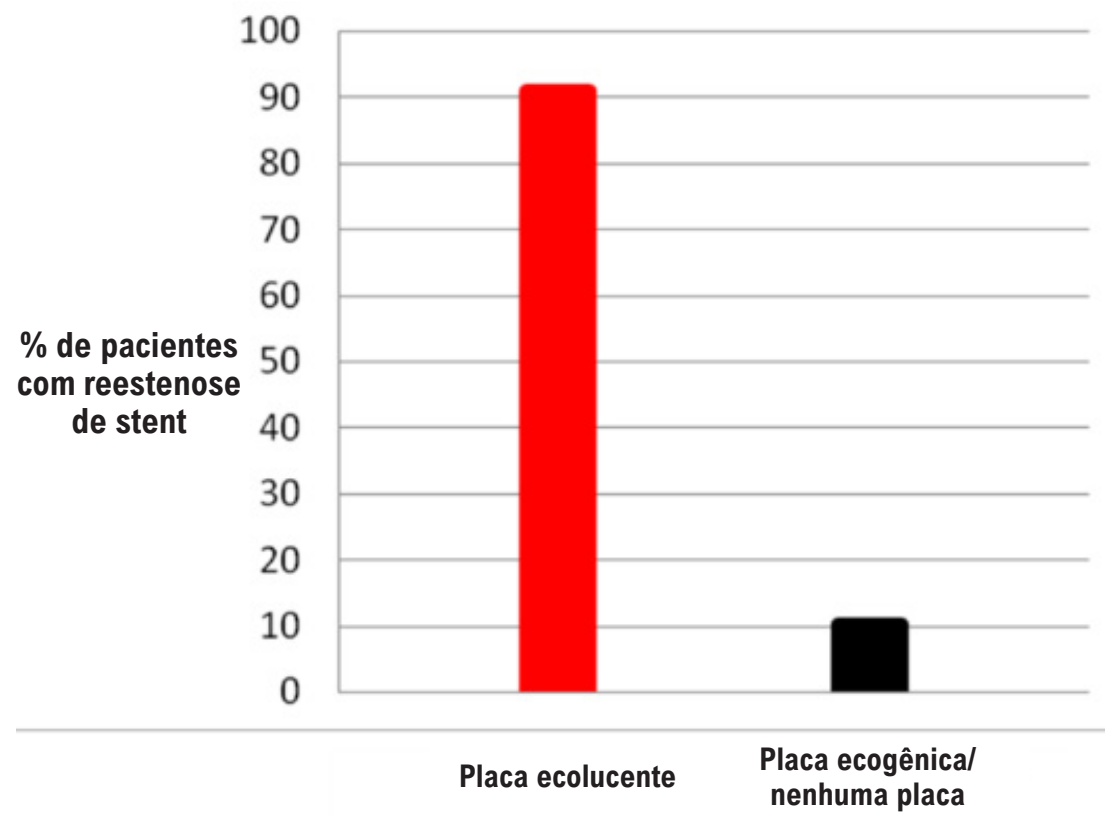

Figura 1 - Porcentagem de pacientes com reestenose de stent coronário nos grupos de pacientes com placa ecolucente e pacientes com placas ecogênicas ou nenhuma placa nas artérias carótidas.

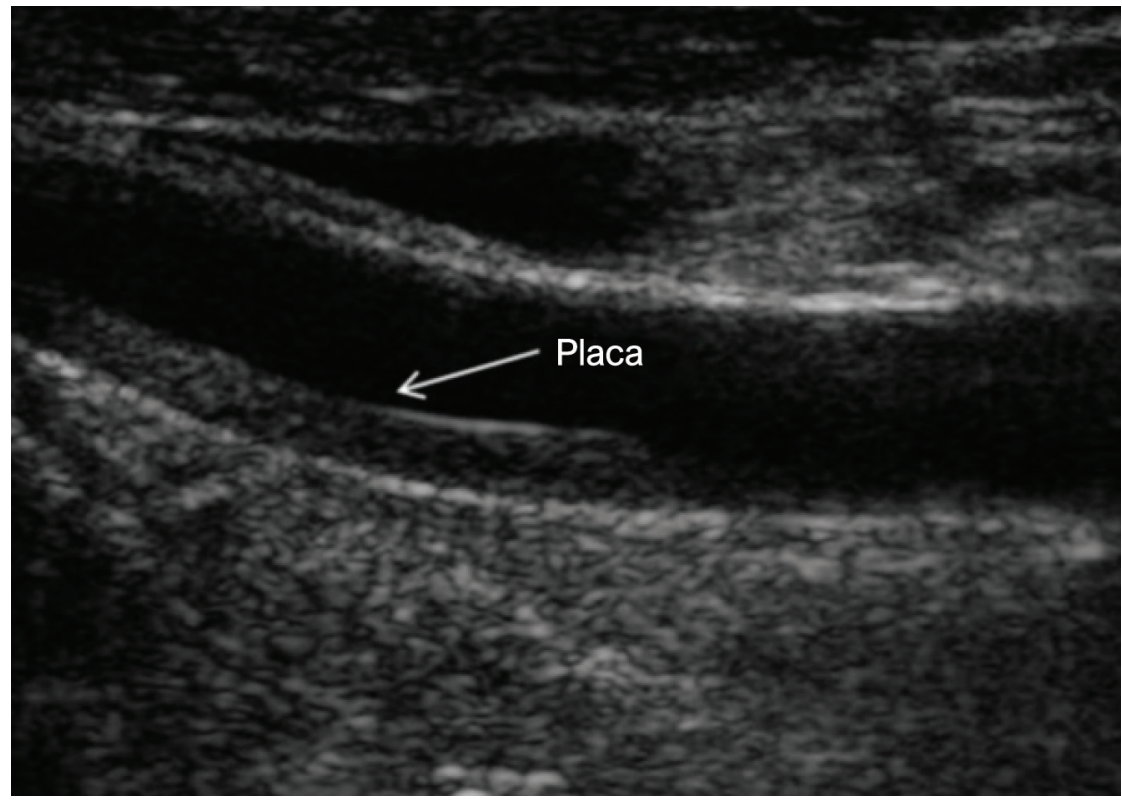

Figura 2 - Imagens ultrassonográfias de placas ateroscleróticas tipo II na artéria carótida esquerda.

foram detectados nas placas ateroscleróticas. ${ }^{22,23}$ As citocinas também estão relacionadas à inflamação aguda e crônica, e sua produção depende em muitos fatores rigorosamente regulados durante a inflamação. Uma ampla variedade de citocinas, tais como TNF- $\alpha$, IL-1, IL-2, IL-3, IL-6, CXCL8, IL-10,
IL-12, IL-15, IL-18, IFN- $\gamma$, M-CSF, TGF- $\beta 1$, TGF- $\beta 2$, e TGF- $\beta 3$, foi detectada nas placas ateroscleróticas. Ainda, em condições de hiperlipidemia, TNF- $\alpha$, IL-1, IL-6, IL-12, IL-15, e IL-18 são produzidos por macrófagos. ${ }^{24}$ Vários estudos sugeriram a hipótese que disfunção endotelial - causada principalmente 


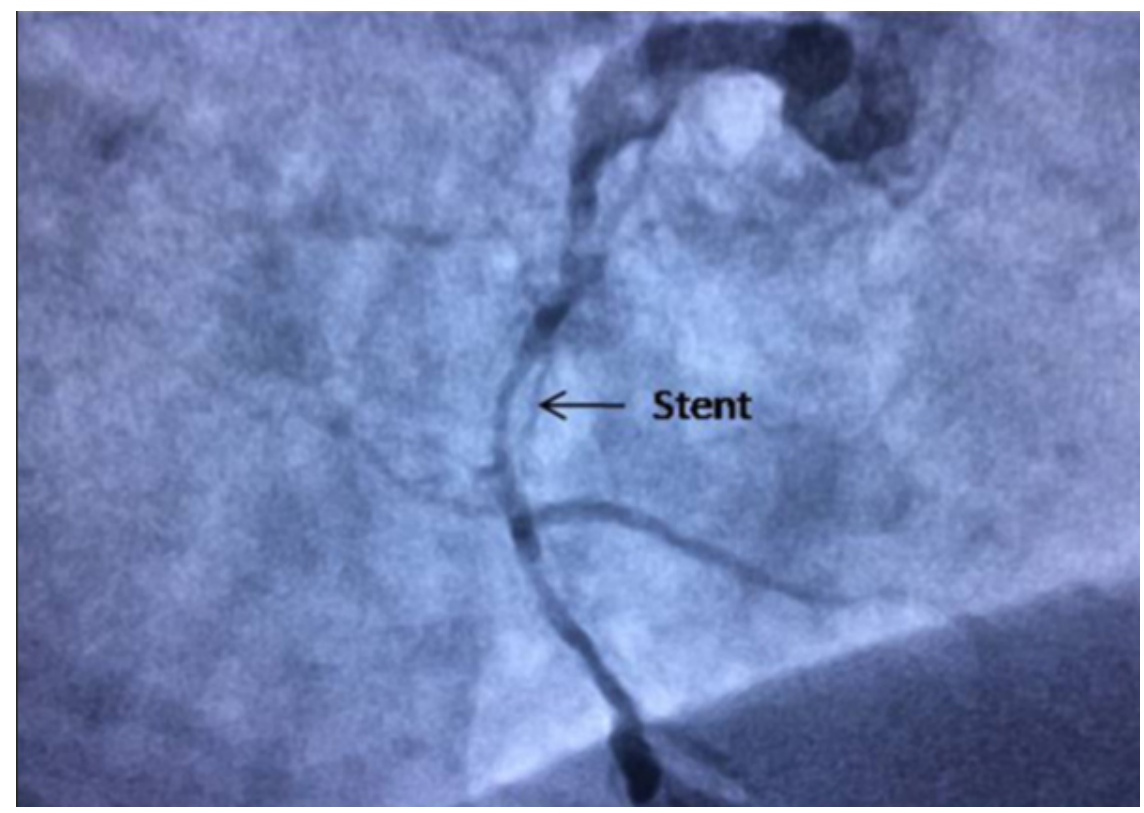

Figura 3 - Achados angiográficos coronários com reestenose de stent na artéria coronária direita.

por níveis elevados de LDL, tabagismo, hipertensão arterial, e diabetes mellitus - é a primeira etapa no desenvolvimento de aterosclerose. Assim, cada etapa da aterosclerose representaria uma diferente fase do processo inflamatório crônico. ${ }^{25}$

As plaquetas também exercem um importante papel no processo aterogênico. Elas podem regular a resposta imune e inflamatória pela secreção de mediadores inflamatórios que modulam o recrutamento de leucócitos aos tecidos inflamados. Plaquetas ativas, as quais expressam P-selectina, foram detectadas em diferentes fases da aterosclerose. ${ }^{26}$

Placas ateroscleróticas ecolucentes - diferente das placas ecogênicas, que contêm mais cálcio e tecido fibroso - são mais ricas em lipídios, elastina, e células inflamatórias, com alta concentração de macrófagos e atividade da metaoloproteinase elevada, os quais exercem importante papel na diferenciação, proliferação e migração celular, e na remodelação vascular. ${ }^{27} \mathrm{~A}$ presença de placa ecolucente na artéria carótida mostrou ser um preditor independente de acidente vascular cerebral e síndrome coronária aguda, incluindo infarto do miocárdio. ${ }^{28,29}$

A reestenose de stent é causada por uma combinação de fatores incluindo denudação endotelial, trauma mecânico, e desarranjo da túnica média e adventícia. Uma reação inflamatória ocorre em estruturas do stent, com infiltração de leucócitos, monócitos, e macrófagos. A gravidade da inflamação é diretamente proporcional ao trauma na parede arterial. Além disso, lesão mecânica da parede do vaso estimula a migração de células musculares lisas (da túnica média) e miofibroblastos (da túnica adventícia) à túnica íntima, onde elas se proliferam. ${ }^{30} \mathrm{~A}$ exposição das túnicas dos vasos facilita o contato com fatores da circulação sanguínea, estimulando a hiperplasia da túnica íntima. Com o passar do tempo, a celularidade diminui e a matriz extracelular começa a predominar na lesão de reestenose. Estudos histopatológicos descrevem uma relação inflamatória mais prolongada após a implantação do stent que após a angioplastia com balão. ${ }^{31}$

Kornowski et al., ${ }^{32}$ relataram que a relação inflamatória da parede arterial nas artérias coronárias de suínos foi frequentemente observada um mês após o implante de stent. A reação inflamatória foi composta principalmente de histiócitos, linfócitos, e formação de granuloma, e de neutrófilos nas formas inflamatórias graves. Houve uma forte correlação entre a extensão da reação inflamatória e a quantidade de formação neointimal no interior dos stents. Segundo os estudos mencionados, que avaliaram os mecanismos de aterogênese e reestenose de stent, a inflamação é um link comum evidente entre placa ecolucente na artéria carótida e reestenose de stent coronário. Ainda, Rothwell et al., ${ }^{33}$ relataram que instabilidade da placa, ou seja, com inflamação, não é meramente um fenômeno vascular local, mas ocorre simultaneamente em vários lugares no leito vascular sistêmico.

Apesar de ser um preditor de doenças cardiovasculares, uma EIM aumentada não elevou o risco para reestenose de stent. Tal fato é consistente com estudos prévios e com o conceito de que o tamanho da placa não contribui tanto como a instabilidade da placa para eventos cardiovasculares. ${ }^{20,34}$ Isso se deve possivelmente ao fato de que o espessamento da camada íntima-média da artéria carótida faz parte do processo de envelhecimento da parede arterial, e não sinônimo de aterosclerose subclínica. Contudo, alterações celulares e moleculares observadas no espessamento da íntima-média estão envolvidas no desenvolvimento e progressão de placas. ${ }^{14}$ Assim, um aumento na EIM sem placas concomitantes não teria relação com os processos inflamatórios na aterosclerose. 


\section{Limitações do estudo}

A validade externa deste estudo é limitada pela avaliação somente de pacientes sintomáticos, diagnosticados com angina estável. No entanto, todos os pacientes neste estudo foram submetidos à angiografia coronária, exame padrão-ouro para o diagnóstico de reestenose de stent coronário, o que aumenta a validade interna do estudo. Outra limitação é o fato de não termos. estudado um grupo de pacientes submetidos ao implante de SF.

\section{Conclusão}

A presença de placa aterosclerótica ecolucente na artéria carótida representa um preditor de risco de reestenose de stent coronário e deveria ser considerada, juntamente com outros preditores de risco, na escolha do tipo de stent a ser implantado na angioplastia coronária.

\section{Contribuição dos autores}

Concepção e desenho da pesquisa: Rodrigues CSA, Nunes HRC, Okoshi K, Hueb JC, Bazan SGZ; Obtenção de dados:
Rodrigues CSA, Reis FM, Silveira CFSMP, Hueb LMS; Análise e interpretação dos dados e Redação do manuscrito: Rodrigues CSA, Bazan R, Reis FM, Silveira CFSMP, Hueb LMS, Carvalho FC, Nunes HRC, Okoshi K, Hueb JC, Bazan SGZ; Análise estatística: Bazan R, Nunes HRC; Revisão crítica do manuscrito quanto ao conteúdo intelectual importante: Bazan SGZ.

\section{Potencial conflito de interesses}

Os autores declaram não haver conflito de interesses pertinentes.

\section{Fontes de financiamento}

O presente estudo não contou com fontes de financiamento externas.

\section{Vinculação acadêmica}

Este artigo é parte de dissertação de Mestrado de Cássia da Silva Antico Rodrigues pela Universidade Estadual Paulista Julio de Mesquita Filho.

\section{Referências}

1. Hoffmann R, Mintz GS, Dussaillant GR, Popma JJ, Pichard AD, Satler LF, et al. Patterns and mechanisms of in-stent restenosis - A serial intravascular ultrasound study. Circulation. 1996; 94(6):1247-54.

2. Cutlip DE, Chauhan MS, Baim DS, Ho KK, Popma JJ, Carrozza JP, et al. Clinical restenosis after coronary stenting: perspectives from multicenter clinical trials. J Am Coll Cardiol, 2002;40(12):2082-9.

3. Karas SP, Gravanis MB, Santoian EC, Robinson KA, Anderberg KA, King SB. Coronary intimal proliferation after balloon injury and stenting in swine: an animal model of restenosis. J Am Coll Cardiol .1992;20(2):467-74.

4. Steele PM, Chesebro JH, Stanson AW, Holmes DR Jr, Dewanjee MK, Badimon L, et al. Balloon angioplasty. Natural history of the pathophysiological response to injury in a pig model. Circ Res. 1985;57(1):105-12.

5. Hill RA, Boland A, Dickson R, Dündar Y, Haycox A, McLeod C, et al. Drugeluting stents: a systematic review and economic evaluation. Health Technol Assess. 2007;11(46):iii, xi-221.

6. Tu JV, Bowen J, Chiu M, Ko DT, Austin PC, HeY, et al. Effectiveness and safety of drug-eluting stents in Ontario. N Engl J Med. 2007;357(14):1393-402.

7. Abbott JD, Voss MR, Nakamura M, Cohen HA, Selzer F, Kip KE, et al. Unrestricted use of drug-eluting stents compared with bare-metal stents in routine clinical practice: findings from the National Heart, Lung, and Blood Institute Dynamic Registry. J Am Coll Cardiol. 2007;50(21):2029-36.

8. James SK, Stenestrand U, Lindbäck J, Carlsson J, Scherstén F, Nilsson T, et al. Long-term safety and efficacy of drug-eluting versus bare-metal stents in Sweden. N Engl J Med. 2009;360(19):1933-45.

9. King SB III, Smith SC Jr, Hirshfeld JW Jr, Jacobs AK, Morrison DA, Williams DO. ACC/AHA/SCAI. Guideline Update for Percutaneous Coronary Intervention. Circulation. 2008;117(2):261-95

10. Hochholzer W, Trenk D, Frundi D, Blanke P, Fischer B, Andris K, et al. Time dependence of platelet inhibition after a 600-mg loading dose of clopidogrel in a large, unselected cohort of candidates for percutaneous coronary intervention. Circulation. 2005;111(20):2560-4

11. Cutlip D, Abbott JD. Clinical use intracoronary bare metal stents. [Cited in 2020 dec 12] Available from: uotodate/com/contents/clinical-use-ofintracoronary-bare-metal-stents
12. Ross R. Atherosclerosis-an inflammatory disease. N Engl J Med 1999;340(2):115-26.

13. Corrado E, Camarda P, Coppola G, Muratori I, Ciaramitaro G, Farinella $M$, et al. Prognostic role of endothelial dysfunction and carotid intimamedia thickness in patients undergoing coronary stent implantation. Int Angiol. 2009;28(1):12-9.

14. Stein JH, Korcarz CE, Hurst RT, Lonn E, Kendall CB, Mohler ER, et al. Use of carotid ultrasound to identify subclinical vascular disease and evaluate cardiovascular disease risk: a consensus statement from the American Society of Echocardiography carotid intima-media thickness task force. Endorsed by the society for vascular medicine. J Am Soc Echocardiogr.2008; 21(2):93-111.

15. Touboul PJ, Hennerici MG, Meairs S, Adams H, Amarenco P, et al. Mannheim Carotid Intima-Media Thickness Consensus. CerebrovasC Dis. $2007 ; 23(1): 75-80$.

16. Lorenz MW, von Kegler S, Steinmetz H, Markus HS, Sitzer M. Carotid intima-media thickening indicates a higher vascular risk across a wide age range: prospective data from the Carotid Atherosclerosis Progression Study (CAPS). Stroke. 2006; 37(1):87-92

17. Gray-Weale AC, Graham JC, Burnett JR, Lusby RJ. Carotid artery atheroma: comparison of preoperative B-mode ultrasound appearance with carotid endarterectomy specimen pathology. J Cardiovasc Surg. 1988; 29(6):676-81

18. Beatt KJ, Serruys PW, Hugenholtz PG. Restenosis after coronary angioplasty: new standards for clinical studies. J Am Coll Cardiol. 1990;15(2):491-8.

19. Califf RM, Ohman EM, Frid DJ, Fortin DF, Mark DB, Hlatky MA, et al. Restenosis: the clinical issues. In: Topol EJ: Text book of interventional cardiology W.B. Saunders, Philadelphia: WB Saunders;1990.p.363-94.

20. Kitta Y, Obata JE, Takano H, Nakamura T, Kodama Y, Fujioka D, et al Echolucent carotid plaques predict in-stent restenosis after bare metal stenting in native coronary arteries. Atherosclerosis 2008;197(1):177-82.

21. Gerrity RG, Naito HK, Richardson M, Schwartz CJ. Dietary induced atherogenesis in swine. Morphology of the intima in prelesion stages. Am J Pathol. 1979;95(3):775-92. 


\section{Artigo Original}

22. Hansson GK, Libby P. The immune response in atherosclerosis: a doubleedged sword. Nat Rev Immunol. 2006; 6(7):508-19.

23. Galkina E, Ley K. Leukocyte influx in atherosclerosis. Curr Drug Targets 2007; $8(12): 1239-48$

24. Tedgui A, Mallat Z. Cytokines in atherosclerosis: pathogenic and regulatory pathways. Physiol Rev 2006; 86(2):515-81.

25. Ross R, Glomset JA. Atherosclerosis and the arterial smooth muscle cell: proliferation of smooth muscle is a key event in the genesis of the lesions of atherosclerosis. Science. 1973;180(4093):1332-9.

26. Von Hundelshausen $P$, Weber C. Platelets as immune cells: bridging inflammation and cardiovascular disease. Circ Res 2007;100(1):27-40.

27. Gronholdt ML, Nordestgaard BG, Bentzon J, Wiebe BM, Zhou J, Falk E, et al. Macrophages are associated with lipid-rich carotid artery plaques, echolucency on B-mode imaging, and elevated plasma lipid levels. J Vasc Surg 2002;35(1):137-45.

28. Polak JF, Shemanski L, O'Leary DH, Lefkowitz D, Price TR, Savage PJ, et al. Hypoechoic plaque at US of the carotid artery: an independent risk factor for incident stroke in adults aged 65 years or older. Cardiovascular Health Study. Radiology. 1998; 208(3):649-54.
29. Honda O, Sugiyama S, Kugiyama K, Fukushima H, Nakamura S, Koide S, et al. Echolucent carotid plaques predict future coronary events in patients with coronary artery disease. J Am Coll Cardiol. 2004;43(7):1177-84.

30. Komatsu R, Ueda M, Naruko T, Kojima A, Becker AE. Neointimal tissue response at sites of coronary stenting in humans: macroscopic, histological, and immunohistochemical analyses. Circulation. 1998; 98(3):224-33.

31. Kleinman ME, Cipolla GD, Cui J, Chronos N, King SBI, Robinson KA. Stent implantation induces late arterial wall cellular proliferation compared to angioplasty in normal rabbits. J Am Coll Cardiol. 1997;29:312A.

32. Kornowski R, Hong MK, Tio FO, Bramwell O, Wu H, Leon MB. In-stent restenosis: contributions of inflammatory responses and arterial injury to neointimal hyperplasia. J Am Coll Cardiol. 1998;31(1):224-30.

33. Rothwell PM, Villagra R, Gibson R, Donders RC, Warlow CP. Evidence of a chronic systemic cause of instability of atherosclerotic plaques. Lancet. 2000;355(9197):19-24.

34. Libby P, Aikawa M. Stabilization of atherosclerotic plaques: new mechanisms and clinical targets. Nat Med. 2002;8(11):1257-62. 